\title{
Aplicación de la metodología de proyecto por los docentes en la enseñanza de educa- ción tecnológica, herramienta fundamental para la reflexión científica-tecnológica ${ }^{1}$
}

Nelson Oyarzún Oyarzún²

Universidad de Los Lagos, Chile ${ }^{3}$

noyarzun@ulagos.cl

1 Artículo de investigación, resultado del trabajo realizado en el seminario doctoral.

2 Doctor en Ciencias de la Educación. Mención Currículo y Didáctica, Universidad De AconcaguaChile

3 Académico 


\title{
Aplicación de la metodología de proyecto por los docentes en la enseñanza de educación tecno- lógica, herramienta fundamental para la reflexión científica-tecnológica
}

\section{Resumen}

El artículo presenta la metodología de proyecto, aplicada en el proceso de enseñanza de las carreras tanto de Pedagogía en Educación General Básica, como de Pedagogía en Educación Básica con Mención en Matemática y Ciencias, en la Universidad de Los Lagos, Chile. El objetivo es mostrar cómo esta metodología genera la reflexión científica-tecnológica de los alumnos. Los resultados obtenidos indican un avance en la reflexión científicotecnológica de los estudiantes de Educación General Básica. En el otro caso solamente se utilizan algunas fases de la metodología de proyecto, lo cual no es suficiente para lograr un buen desarrollo de la reflexión científicatecnológica. Al observar las clases se descubrieron características propias de la metodología tradicional conductista, tales como conducción reiterada y exposición oral del profesor. La Metodología estudiada ofrece elementos necesarios para innovar y mejorar la práctica pedagógica. Entre ellas, la reflexión respecto de situaciones problemáticas, el análisis de estas, la búsqueda de información y la constante evaluación de las respuestas más adecuadas al problema presentado.

Palabras clave: Metodología de proyecto, reflexión científica-tecnológica, innovación práctica pedagógica.

\section{Implementation of a Project Methodology in teaching technology education, a fundamental tool for scientific-technological reflection}

\begin{abstract}
The article presents a project-methodology applied to teaching all academic programs in Basic Education as General Education in Basic Education with a minor in Mathematics and Science at the Universidad de Los Lagos, Chile. The aim is to show how this methodology generates a scientific-technological reflection of students. The results obtained indicate two technological progresses in scientific thinking of students in General Basic Education. Contrasted with a case whereby only some phases of the project-methodology were used, there was no enough exposure to yield a successful development of scientific-technological reflection. While observing classes, specific characteristics proper to traditional behaviorism were discovered as exemplified in a repeated behavior of oral exposure to the teacher. This project-based-methodology-study informs innovation and improvement with necessary elements to the teaching expertise. Among these necessary elements is the reflection on problematic situations, their analysis, the search for information and the stanch assessment of the most appropriate responses to the problem under treatment.
\end{abstract}

Key words: Project methodology, scientific-technological, innovation, reflection, pedagogical practices.

\section{Aplicação da Metodologia de Projeto pelos docentes no ensino da educação tecnológica, ferra- menta essencial para a reflexão científica-tecnológica.}

\section{Resumo}

O artigo apresenta a Metodologia de Projeto aplicada no processo de ensino das carreiras de Pedagogia na Educação Geral Básica e Pedagogia na Educação Básica com Menção em Matemática e Ciências da Universidad de Los Lagos, Chile. O objetivo é de apresentar como essa metodologia gera a reflexão científica-tecnológica dos alunos. Os resultados obtidos assinalam um avanço na reflexão científica-tecnológica dos estudantes de Educação Geral Básica. No outro caso, somente são usadas algumas frases da Metodologia do Projeto, o qual não é suficiente para atingir um bom desenvolvimento da reflexão científica-tecnológica. Ao observar as aulas foram encontradas características próprias da metodologia tradicional behaviorista, tal como a condução reiterada e a exposição oral do docente. A Metodologia analisada oferece elementos necessários para inovar e melhorar a prática pedagógica. Entre eles, a reflexão com relação a situações problemáticas, a análise delas, a busca de informação e a avaliação constante das respostas mais adequadas ao problema apresentado.

Palavras chave: Metodologia de Projeto, reflexão científica-tecnológica, inovação, práticas pedagógicas. 
Aplicación de la metodología de proyecto por los docentes en la enseñanza de educación tecnológica, herramienta fundamental para la reflexión científica-tecnológica

\section{Introducción}

La metodología de proyecto, en el entorno chileno, se incorporó al modelo curricular desde la década de los noventas, a través de la Educación Tecnológica respondiendo a una necesidad para conseguir una educación de calidad, esta idea cobra mucha fuerza producto de los avances científicos -tecnológicos y de la sociedad en los últimos años. Más tarde, esta metodología pasa a ser un tema transversal utilizado en las distintas asignaturas del currículo, especialmente en ciencia y tecnología. (Branda, 2008). Sin embargo, la Educación Tecnológica, incorpora esta metodología para la construcción del conocimiento a partir de un hacer reflexivo; para permitir a los alumnos desarrollar conocimientos, habilidades, aspectos científicos y éticos. Apunta a resolver problemas de conocimientos más cercanos y reales, en el mundo científico y tecnológico con los que el alumno debe convivir y debe enfrentar diariamente.

Para resolver la problemática anterior nacen distintos programas formativos para iniciar la apropiación curricular. Sin embargo, en la actualidad conviven básicamente, el modelo de racionalidad pedagógica que entiende la capacidad intelectual y el área afectiva del discente como dos instancias separadas (Pavié, 2011). En consecuencia, coexisten en los programas rasgos caracterizadores de distintos modelos y épocas. Por un lado, el modelo de formación conductista (ListonZeichner, 1991) que está muy arraigado en el entorno pedagógico a pesar de su antigüedad, y que concibe la enseñanza como una actividad con base científica y a los profesores como profesionales competentes. Por otro lado, el modelo técnicoacademicista (Pérez Gómez, 1998) que versa sobre el desarrollo del conocimiento experto y las consecuencias "positivas" que debería tener en el desarrollo profesional.

A lo anterior, tal vez, se agrega un tercer componente de los programas de formación que responde a características de un modelo centrado en el proceso (Ferrero, 1983), a partir de la entrada del enfoque constructivista y de los enfoques cognitivos del aprendizaje en la década de los noventa a causa de la Reforma Curricular. (Abraham, Marta, 2001). Pero decimos tal vez, porque sería un rasgo que solo se considera parcialmente en los programas formativos, debido a que no existe una apropiación curricular efectiva y completa de los programas de la enseñanza obligatoria de estas nuevas directrices que el enfoque constructivista implica. Esta dificultad está refrendada por lo que afirma Pinto, 2001, en cuanto a que las políticas y acciones de las reformas educativas llegan de manera desintegrada a la unidad académica, fortaleciendo con ello un estilo de formación fragmentaria e individualista.

Además, existen causas que hacen de la enseñanza y aprendizaje de las matemáticas un proceso tedioso y poco significativo, como por ejemplo: cuando 
no existen en el aula los medios para este fin; los espacios para la interacción entre docente y estudiantes, a lo largo del desarrollo de las temáticas, no son los adecuados; los recursos didácticos no son pertinentes o no se cuenta con ellos en el momento oportuno; las pedagogías y metodologías con las que se desarrolla la clase no están en consonancia con los objetivos trazados para la misma; los aportes de la evaluación al aprendizaje de las matemáticas son escasos o nulos, entre otras. Entonces, el articular de forma reflexiva este conjunto de elementos de la educación, pueden llegar a convertir el aula de clase en un verdadero recinto donde se construya conocimiento de una forma dinámica, libre y en continua relación con el medio. De esta forma, el aprendizaje de las matemáticas pueda convertirse en eje fundamental, que potencie habilidades, capacidades y competencias en los estudiantes, de modo que reconozcan su importancia a partir de la utilidad práctica (D'Amore et ál., 2008), y logren una trascendencia del conocimiento aprendido y construido en el aula más allá de los muros institucionales.

La importancia del presente trabajo radica en estudiar una alternativa de aprendizaje para los alumnos en los procesos científicos- tecnológicos, y cómo la metodología de proyecto, adquiere importancia en la consecución de las reflexiones científicas. Esto radica en que, por un lado, la metodología tiene entidad propia como estrategia para abordar tareas metodológicas y por otro lado, es contenido centralizador, organizador y vertebrador de otros aprendizajes en las ciencias y otras áreas del conocimiento.

\section{Conceptos centrales}

\section{Tecnología}

La tecnología se ha transformado en un componente de en la cultura que provoca admiración y ha llegado a ser casi un estilo de vida, la mayoría de los aparatos que nos rodean no existían hace 50 años, dependemos de estos productos, pero sabemos muy poco acerca de ellos. Admiramos dispositivos que nos parecen milagrosos y nos sorprende que en muchos sistemas educativos, hoy día, la tecnología aún no haya sido objeto de estudio para las nuevas generaciones. Conviene entonces preguntarse: ¿qué entendemos por tecnología? En una acepción muy elemental podemos decir que: "[...] es la manera de hacer las cosas [...]", como lo señala, (Ferreira, C., Gil-Pérez, D. y Vilches, A. 2006) pero la definición de tecnología aún no logra un consenso unánime, e incluso, está sujeta a variantes lingüísticas.

La tecnología-saber cómo y por qué hacer, persigue desarrollar soluciones prácticas a problemas y necesidades existentes, de un modo sistemático y ordenado. Desde el punto de vista etimológico la definimos como estudio y reflexión sobre técnicas. 
Aplicación de la metodología de proyecto por los docentes en la enseñanza de educación tecnológica, herramienta fundamental para la reflexión científica-tecnológica

| Nelson Oyarzún Oyarzún |

Toda técnica comporta el proceso de saber-poder-hacer, pero a su vez está asociada a un amplio conjunto de conocimientos, integrado por los de tipo operativo (saber cómo hacer) propio de la técnica, y con los fundamentos científicos (Branda, 2008).

Asimismo, Branda (2008) se refiere a la ciencia y tecnología, indicando que: "[...] la primera descubre o desvela las leyes que gobiernan la naturaleza, y la tecnología utiliza esos conocimientos para su transformación [...]". Según este esquema, la presencia de conocimientos científicos en el origen del desarrollo tecnológico sería lo que diferenciaría a la actividad tecnológica (fundamentada en conocimientos científicos), de la mera técnica artesanal en que el conocimiento experimental no existe. Estas prácticas solo se basan en las intuiciones y en la experiencia pasada de sucesivas pruebas por ensayo y error.

Partiendo de esta idea, se considera con frecuencia que la actividad tecnológica organiza su enseñanza distinguiéndose con claridad dos fases bien diferenciadas: la más teórica, que corresponde al análisis y diseño conceptual del artefacto, y la de su producción y realización práctica. Muchas veces en la enseñanza de la tecnología se ha insistido en la importancia de distinguir ambas fases y de conceder gran importancia a la primera, a fin de sortear el riesgo que la enseñanza de la tecnología pueda reducirse a las técnicas artesanales. Según esta idea, en el aula de tecnología el papel del docente y de los alumnos dentro de ella no deben ser réplicas del taller del artesano y de la relación entre este y el aprendiz. Como señala Branda (2008): "[...]el aprendiz aprende del artesano ayudándole, y copia sus maneras de actuar y desarrolla las destrezas propias del artesano [...]". Pero, ese no debe ser el modelo del aula de tecnología, porque de ser así, la Educación Tecnológica se reduciría a la enseñanza de las artesanías tradicionales, lo que dejaría de nuevo al docente de tecnología en un lugar secundario respecto a los enseñantes de las disciplinas académicas, y a la tecnología en un lugar marginal del currículo de las instituciones educativas.

Según este tópico, el aula-taller de tecnología no debe tomar sus referentes de las técnicas artesanales sino de las tecnologías industriales, y parecerse a un espacio de construcción de prototipos diseñados previamente de forma más científica y conceptual. En esta idea de la enseñanza de la tecnología, el aula-taller y el aula ordinaria de tecnología mantienen entre sí una relación análoga a la que se da entre el laboratorio y el aula ordinaria en la enseñanza de las ciencias. En el aula ordinaria se enseñan, y supuestamente aprenden, los aspectos conceptuales de ambos tipos de disciplinas, las teorías, los principios, las leyes, en el caso de la enseñanza de las ciencias; los problemas planteados y los cálculos para su resolución. Además, el diseño con lápiz y papel de posibles prototipos en el caso de la enseñanza de la tecnología. "En el laboratorio y en el aula-taller se desarrollan los momentos prácticos de las ciencias y las tecnologías, en el primero se recrean los procedimientos experimentales que probarían la verdad de lo aprendido en el aula ordinaria, en el segundo, se simulan los procesos prácticos de construcción de los artefactos diseñados sobre el papel [...]" 
(Gutiérrez, 2002). Así mismo, Ginestié (2001) también se refiere a las aulas prácticas, en el sentido: "[...] que sean laboratorios de ciencia o taller de tecnología; son más entretenidos para los alumnos que las teóricas [...]", lo importante es producir un equilibrio necesario entre la teoría y la práctica.

\section{La educación tecnológica en Chile, y en pedagogía básica}

En Chile, la Educación Tecnológica se incorporó como plan piloto, con el nombre de: área de la tecnología. Proyecto: "Iniciación a la vida del trabajo y la creatividad" en el año 1990, enfocado al segundo ciclo de Educación General Básica. Este plan de desarrollo consistía en capacitar a profesores en dos direcciones: la primera, en el desarrollo curricular del Proyecto Educativo Institucional y la segunda, calificar profesores para enfrentar a la llamada área de tecnología. Estas capacitaciones fueron realizadas en Santiago, en el CPEIP ${ }^{4}$. En cuanto al material de apoyo del proyecto, este consistió en aulas tecnológicas de implementación española. Las aulas tecnológicas incorporaban materiales didácticos que permitían a los alumnos realizar el diseño y producción de prototipos, además mecanismos y circuitos, y algunos conceptos de gestión tecnológica. De esta manera, se hacía una primera aproximación a la definición del sector de tecnología en nuestro país.

En la Universidad De Los Lagos, la educación tecnológica es incorporada en 1999, en los primeros años de los estudiantes de pedagogía en educación general básica. Más tarde, en cada semestre de la carrera mencionada se incluyó esta asignatura. En la actualidad, además de pedagogía en educación general básica, también se incorporaron pedagogía básica con mención en matemática, y ciencias de la naturaleza, asimismo, educación parvularia, también se suma a esta nueva área del conocimiento.

\section{Didáctica de la educación tecnológica}

De las Bases Curriculares (2013) del Ministerio de Educación de Chile, se desprende el enfoque y las orientaciones didácticas propias de la Educación Tecnológica. La metodología de esta asignatura se apoya en el desempeño de los alumnos y alumnas en las siguientes dimensiones: el hacer, la reflexión sobre los procesos que conforman ese hacer, en los conocimientos, habilidades y actitudes en la resolución de problemas que signifiquen un "hacer tecnológico". Como se manifiestan en los programas de estudios de Educación Tecnológica, del Ministerio de Educación de Chile.

4 Centro de Experimentaciones e Investigaciones Pedagógicas de Chile. Este Centro depende del Ministerio de Educación, en este lugar se realizan cursos para los profesores, además tiene a cargo la regularización de los distintos perfeccionamientos que entregan las Universidades a nivel nacional. 
Aplicación de la metodología de proyecto por los docentes en la enseñanza de educación tecnológica, herramienta fundamental para la reflexión científica-tecnológica

Este enfoque didáctico propicia la participación activa de los alumnos y alumnas en todos los ámbitos definidos a partir de los ejes, en actividades que permitan usar una variedad de medios para distinguir y anunciar problemas y resolver problemas prácticos en un contexto social. Además, adquirir y usar durante su trabajo tres tipos de habilidades interrelacionadas: el cómo hacer, la comprensión de procesos y la adquisición de conocimientos, a través de metodología propia del sector. Según Famiglietti, y María, (2001) esto implica, por parte del profesor, arriesgarse a tomar opciones, desarrollar múltiples soluciones a problemas, probar y mejorar, prevenir, trabajar en grupo en forma colaborativa, responsabilizarse por los resultados y administrar los recursos en forma efectiva y eficiente.

\section{Metodología de proyecto y sus características}

Los proyectos de aulas se han tomado como una estrategia didáctica para desarrollar la práctica pedagógica de la Educación Tecnológica. Las razones para ello son múltiples y van desde verlos como un proceso de investigación, hasta la producción de un producto. Sin embargo, no existe claridad de los profesores para trabajar con esta metodología de proyecto, no cualquier cosa que se trabaje en el aula es un proyecto (Ginestié, 2001, 55).

El mismo Ginestié (2001) sostiene que "[...] el proyecto de aula en Educación Tecnológica es un conjunto de actividades y tareas de carácter tecnológico, científico, lúdico, técnico, artístico, etc. Que se programa para alcanzar un propósito, surge como respuesta un proceso de análisis y reflexión sobre la realidad y busca solución a problemas y/o necesidades reales, en un contexto determinado [...]". En consideración a lo anterior, una de las características de la metodología apunta al nacimiento de un proyecto de aula de un área específica. A partir de una construcción colectiva y atendiendo a las necesidades, intereses e inquietudes de los participantes y en su desarrollo se involucra a las demás áreas, pues requiere de todo el apoyo logístico académico y técnico que sea posible para llegar a feliz término.

Frente a las características de la metodología es necesario plantear los problemas en el aula de tal manera que sean posibles de solucionar. Produciendo un refuerzo positivo en los alumnos, como propone (López, 2001). Así la solución debe estar al alcance del nivel de desarrollo y capacidad de los alumnos. La búsqueda de esa solución llevará implícita una serie de dificultades, cuya superación generará aprendizajes. El grado de interés del alumno desempeñará un papel determinante. Ciertamente, dependerá del profesor el hacer un planteamiento que pertenezca al centro de atención del alumno. En esencia, este método y en el trabajo en Educación Tecnológica se debe tener espacio y tiempo para aprender y enseñar, esto sucederá en la medida en que el profesor se involucre en el desarrollo de las actividades de sus estudiante y depende de la ayuda que podamos brindarles a los alumnos para desarrollar la comprensión y conocimiento del diseño y la tecnología. Potenciar 
sus capacidades individuales y colectivas, que aprendan a sortear sus dificultades, planeando diferentes alternativas para poder superarlas. Así, la práctica del profesor debe implicar una gran cantidad de actividades que permitan a los niños y niñas involucrarse dentro de ella.

El profesor deberá tener claridad en su papel de enseñanza durante el trabajo para desarrollar las competencias necesarias y que no marginzarse al intervenir en el trabajo de los niños, en algunas ocasiones tutelando en otras proponiendo: "Todos los aprendices, adultos y niños aprenden mejor cuando ven la necesidad de aprender", no hay un control único según Ackerman (cit. en Ferrero1998: 85). Los niños que trabajan en un proyecto a menudo encuentran que hay tareas que quieren hacer, pero, que no saben cómo. En un medio adecuado, ellos deberán ser capaces de solicitar la guía del profesor, de esta manera, sus aprendizajes deben ser más efectivos al identificar sus necesidades.

"La metodología de proyecto consiste en una estrategia de enseñanza, es un modelo donde los alumnos planean, implementan y evalúan proyectos que tienen aplicación en el mundo real más allá de las aulas" (Ferrero, 1998). En ella, se recomiendan actividades de enseñanzas interdisciplinarias, de largo plazo y centradas en el estudiante, en vez de lecciones cortas y aisladas. Las estrategias basadas en proyectos, tienen sus raíces en la aproximación constructivista que evolucionó a partir de los trabajos de psicólogos como: Vigotsky, y Dewey. (Cit. en Ferrero 1998: 93). La metodología de proyecto ha sido primero asunto de algunas corrientes activas. El proyecto se inscribe en una oposición a una escuela tradicional, centrada en el aprendizaje memorístico. Hablar de la estrategia de proyecto presenta ciertas ventajas. Trabajar con estas metodologías incluye trabajar en forma integrada, y no ver los contenidos en forma aislada.

La metodología de proyecto aplicada a nivel Universitario puede apuntar a los siguientes objetivos:

1. Lograr movilizar los saberes o procedimientos, desarrollar capacidades, y descubrir nuevos saberes científicos-tecnológicos y nuevos mundos, en una perspectiva de sensibilización y de motivación.

2. Plantear obstáculos que puedan ser sorteados a partir de nuevos aprendizajes, que deben alcanzarse fuera del proyecto. Además de provocar nuevos aprendizajes en el marco del mismo proyecto, incrementando la cooperación y la inteligencia colectiva.

3. Ayudar a cada alumno a tomar confianza en sí mismo, forma de emprendimiento, de toma de un poder de actor. Asimismo, desarrollar la capacidad de autoestima, y la capacidad de hacer acciones y negociarlas. Formar para la concepción y la conducción de proyectos. 
Aplicación de la metodología de proyecto por los docentes en la enseñanza de educación tecnológica, herramienta fundamental para la reflexión científica-tecnológica

"En la implementación de las metodologías es muy importante que los involucrados o interesados tengan claridad sobre los objetivos del proyecto y su metodología para que sean planificados e incorporados de manera efectiva" (Cajas, 2001). La metodología de proyecto debe ponerse en funcionamiento en forma secuencial, con complejidad creciente, y desde la praxis, finalizando con la reflexión científica-tecnológica, y teórica. Esto sin duda es muy importante para que los alumnos adquieran conocimientos significativos, duraderos, pertinentes y para toda la vida. La actividad científica-tecnológica requiere de propuestas novedosas, que tengan una aplicación real. Las fases de la metodología de proyecto son las siguientes:

a). Planteamiento del problema o necesidad: el planteamiento del problema es introducir al alumno en una necesidad a solucionar. La metodología de proyecto en ciencia y tecnología es un conjunto de actividades y tareas de carácter tecnológico, científico, lúdico, técnico, artístico, etc. eue se programan para alcanzar un propósito, surge como respuesta a un proceso de análisis y reflexión sobre la realidad y busca dar solución a problemas y/o necesidades reales, en un contexto determinado como el hogar, el colegio, la comunidad, la industria, el campo, etc.

b). Búsqueda de información, investigación, análisis, experimentación: cuando el problema no tiene solución clara o dispone de varias soluciones, necesitamos información. Esta información hay que definirla, buscarla y procesarla en cuanto a la necesidad de centros de información (bibliotecas, empresas, revistas, gentes para entrevistas, computación, catálogos, etc.) Mediante el análisis conseguimos información contextualizada reflexiva de un objeto en concreto y/o sistema. Esta fase es de suma importancia porque es el momento en que el profesor desarrolla, estimula la reflexión científica tecnológica a partir del análisis y la experimentación de sistemas tecnológicos.

c). Diseño, planificación y construcción (producción): dentro de la metodología de proyecto, tras plantear el problema, se ha de diseñar. El alumno tiene la mayoría o parte de información necesaria para llevar a cabo el proyecto. El diseño de un objeto o mecanismo es una tarea compleja, tiene que ser abordada desde distintas perspectivas: formas, función, funcionamiento, materiales, coste, etc.

d). Evaluación del proyecto (ifunciona?): Este es el momento en el que el objeto diseñado es una realidad, valorar el resultado obtenido tiene especial significado. Hay que analizar la eficacia del objeto realizado.

e). Memoria y exposición, comunicación (síntesis de los procesos científicos-tecnológicos): si bien el profesor centrará su actuación en analizar los aspectos que considere relevantes según los objetivos del área, el alumno realizará una memoria descriptiva donde recoja todo el proceso seguido. 
Hoy en Chile, el Programa de Estudios de Educación Tecnológica (2013), del Ministerio de Educación, incorpora la metodología de proyecto para provocar un cambio radical de la práctica educativa. Se señala en el mismo programa: "Es un cambio en la manera de enseñar, en que el profesor deja de ser el que posee todo el conocimiento, para transformarse en un mediador, que desarrolla en los estudiantes las competencias necesarias para apropiarse del conocimiento por sí mismos".

Hoy en Chile, el Programa de Estudios de Educación Tecnológica (2013), del Ministerio de Educación, incorpora la metodología de proyecto para provocar un cambio radical de la práctica educativa. Se señala en el mismo programa: "Es un cambio en la manera de enseñar, en que el profesor deja de ser el que posee todo el conocimiento, para transformarse en un mediador, que desarrolla en los estudiantes las competencias necesarias para apropiarse del conocimiento por sí mismos".

Respecto a lo anterior, Muñoz (2009 pp. 101-126) señala que se está discutiendo como la metodología de proyecto puede utilizarse en áreas del conocimiento del medio, que asegure aprendizajes significativos y funcionales en los alumnos. Para lograr estos aprendizajes, esta metodología realiza un enfoque globalizador, relacionando cada contenido en forma activa con el conocimiento de la realidad.

Juana Coria (2009 pp.1), en su artículo, El Aprendizaje por Proyectos, señala que esta metodología de proyecto dentro del currículo no es un tema nuevo, sin embargo, la metodología del aprendizaje por proyectos es diferente, puesto que está centrada el trabajo entre profesores y alumnos como conjunto, lo cual desarrolla en ambos habilidades tecnológicas y de aprendizaje colaborativo.

Bradbury (2010), propone la necesidad de incluir en el currículo la Educación Tecnológica, empezando por la formación y la capacitación formativa de los docentes, (en metodologías y otras materias) para luego involucrar a todo el estudiantado de todos y cada uno de los diferentes niveles educativos (desde preescolar hasta la educación superior). Asimismo, López, 2001 manifiesta que la metodología de proyecto es una forma de dar respuestas a la sociedad en la formulación de problemas y recursos educativos.

Respecto de la formación del profesorado,, Nikel (2014) señala que el profesorado universitario en su formación de metodologías activas de enseñanza debería desarrollar la metodología de proyecto. En efecto, en los últimos años algunas universidades europeas, como Aalborg y Maastricht, entre otras, están transformando la enseñanza tratando de centrarla en el estudiante, dirigirla al desarrollo de competencias, una preocupación no solo por "qué se aprende", sino también por "cómo se aprende". Afirma que gran parte de la enseñanza universitaria se centra en la transmisión de la información, observándose graves carencias en aspectos tan importantes como implicar a los estudiantes en un proceso de enfrentarse con la incertidumbre, la profundización en los significados y la resolución de problemas. 


\section{Metodología}

\section{Diseño y objetivos}

La presente investigación se adscribe a un enfoque cuantitativo y diseño experimental, para establecer el efecto de la variable reflexiones científicas-tecnológicas y metodología de proyecto. Así es como se realizan grupos de medición antes, pre test y medición después, post test. Es de carácter descriptivo explicativo, según Briones (1999), porque busca especificar las características del grupo que se sometió al análisis a través de la recolección de datos, mediante los cuales se pudo describir y explicar el estudio. De esta manera, la información referente a la reflexión científicatecnológica de los alumnos, se recaba a través de hojas de respuestas estructurada a partir de imágenes (dibujos) representadas con razonamientos científicostecnológicos, dirigidos a los estudiantes de pedagogía en educación general básica, básica con mención en matemática y ciencias de la naturaleza. En cuanto a los profesores dan respuesta a un cuestionario estructurado, respecto de la variable metodologías usadas en las clases de educación tecnológica.

A continuación se exponen los objetivos generales de la investigación:

1) Establecer el grado de reflexión científica-tecnológica, de los alumnos de Pedagogía en Educación General Básica y Pedagogía Básica con mención en matemática y ciencias de la naturaleza, en la Universidad de los Lagos de Osorno.

2) Identificar las características de la metodología de proyecto, aplicada por los docentes del las carreras de Pedagogía General Básica con mención en matemática y ciencias de la naturaleza en la Universidad de los Lagos de Osorno.

\section{Muestra}

La muestra fueron los alumnos de sexto semestre, año 2014, de Pedagogía en Educación General Básica y Pedagogía Básica con mención en matemática y ciencias de la naturaleza de la Universidad de los Lagos de Osorno, Chile. De esta manera, se trabajó con un total de 60 alumnos y 5 profesores que realizaron clases en estas carreras.

\section{Variables de investigación e instrumentos}

En este sentido, las variables de esta investigación fueron instauradas para ser medibles y ver cómo influyen en los resultados que se estudiaron con profundidad respecto de las preguntas de investigación. Las variables son: metodología de proyecto aplicada 
por los profesores y reflexión científica-tecnológica de los alumnos, de las carreras mencionadas. Los datos de la investigación fueron recogidos a través de dos encuestas estructuradas y experiencias científicas - tecnológicas dirigidas a los alumnos. El tipo de datos fue de carácter cuantificado, mostrando la relación que existe entre dos o más variables. Los criterios fueron señalados en competencias científicas centradas en el conocimiento científico y en la utilización del mismo. Estos hacen posible actuar e interactuar de manera significativa en situaciones en las cuales se requiere reflexionar, explicar y aplicar comprensivamente los conocimientos científicos, como en el caso de resolver problemas de naturaleza científica y tecnológica, así como en el de analizar críticamente la forma en que ciencia y tecnología influyen en el modo de vida de la sociedad actual.

El análisis de datos se presenta en una dimensión cuantitativa. Se podrá estimar el enfoque de las variables, en cuanto a la visión que tienen los alumnos respecto a las clases realizadas con la metodología de proyecto y su importancia en el desarrollo científico- tecnológico.

Para medir la opinión de alumnos respecto del ítem seleccionado, se creó una variante de la escala tipo Likert, denominada escala de apreciación, y citando a Hernández (1999), esta escala numérica permite dar mayor facilidad al manejo de los datos y subsecuentes análisis del contenido efectuado por medio de la codificación. En este caso, se utilizaron enunciados con experiencias metodológicas y de reflexión científica-tecnológica que debieron ser validados por tres académicos de la Universidad De Los Lagos.

Para establecer el grado de reflexión científica-tecnológica que alcanzaron los alumnos al final del proceso de este estudio realizado, se establecieron los criterios científicos-tecnológicos señalados en la tabla 1, así mismo, incorporando los criterios utilizados por los profesores, respecto de la metodología de proyecto definido en la misma tabla.

TABLA 1. Propuesta de reorganización de dimensiones, ejes y aspectos a desarrollar del Lineamiento Pedagógico y Curricular del Distrito del Bogotá - 2013.

\begin{tabular}{|c|c|c|}
\hline Metodología de proyecto & Proceso científico & Proceso tecnológico \\
\hline Presentación del problema. & Fenómeno natural. & Determinar la necesidad. \\
\hline $\begin{array}{c}\text { Análisis del problema. } \\
\text { Diseñar y considerar } \\
\text { soluciones alternativas. }\end{array}$ & Describir el problema. & $\begin{array}{c}\text { Analizar y describir el } \\
\text { problema. }\end{array}$ \\
\hline Elegir y evaluar la solución. & Seleccionar hipótesis. & $\begin{array}{r}\text { Seleccionar y solucionar la } \\
\text { necesidad. }\end{array}$ \\
\hline
\end{tabular}


Aplicación de la metodología de proyecto por los docentes en la enseñanza de educación tecnológica, herramienta fundamental para la reflexión científica-tecnológica | Nelson Oyarzún Oyarzún |

TABLA 2. Rangos para categorizar las respuestas de los alumnos

\begin{tabular}{|c|c|c|}
\hline Nivel bajo de reflexión & $\begin{array}{c}\text { Nivel medio de } \\
\text { reflexión }\end{array}$ & Nivel alto de reflexión \\
\hline $\begin{array}{c}49 \% \text { y menos de las } \\
\text { respuestas acertadas, en las } \\
\text { experiencias científicas- } \\
\text { tecnológicas. }\end{array}$ & $\begin{array}{c}50 \text { a } 69 \% \text { de las } \\
\text { respuestas acertadas } \\
\text { en las experiencias } \\
\text { científicas- } \\
\text { tecnológicas }\end{array}$ & $\begin{array}{c}70 \% \text { y más de las } \\
\text { respuestas acertadas en las } \\
\text { experiencias científicas- } \\
\text { tecnológicas. }\end{array}$ \\
\hline
\end{tabular}

TABLA 3. Rangos para categorizar las respuestas de los profesores

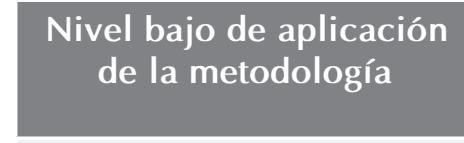

$49 \%$ y menos de las respuestas señaladas por los profesores.

\section{Nivel medio de aplicación de la metodología}

50 a $69 \%$ de las respuestas señaladas por los profesores.
Nivel alto de aplicación

de la metodología

$70 \%$ y más de las respuestas señaladas por los profesores

\section{Procedimientos para el análisis de datos}

La recogida de datos se llevó a cabo en forma grupal durante las horas de Educación Tecnológica. El encuestador informó a los profesores y alumnos acerca de los objetivos de la investigación mediante una carta, y se solicitó el consentimiento para su aplicación. No se estableció límite de tiempo para las respuestas. De esta manera, el análisis de los datos se realizó primero en forma manual, luego se utilizó el programa estadístico SPSS 19 y las técnicas principalmente fueron estadística descriptiva de tendencia central (media) y dispersión (desviación típica), cálculos de frecuencia y porcentajes. También se incorporó el programa Excel, para realizar los gráficos.

\section{Resultados}

A continuación se presenta el análisis realizado, desde el cual se obtienen los resultados de las unidades de análisis elaboradas. Los datos obtenidos están en coherencia con los objetivos planteados en este estudio. El siguiente análisis se llevó a cabo desde los objetivos planteados: reflexión científica-tecnológica de los alumnos y de metodología de proyecto usada por los profesores. 
Gráfico 1. Reflexión científica y tecnológica de los alumnos

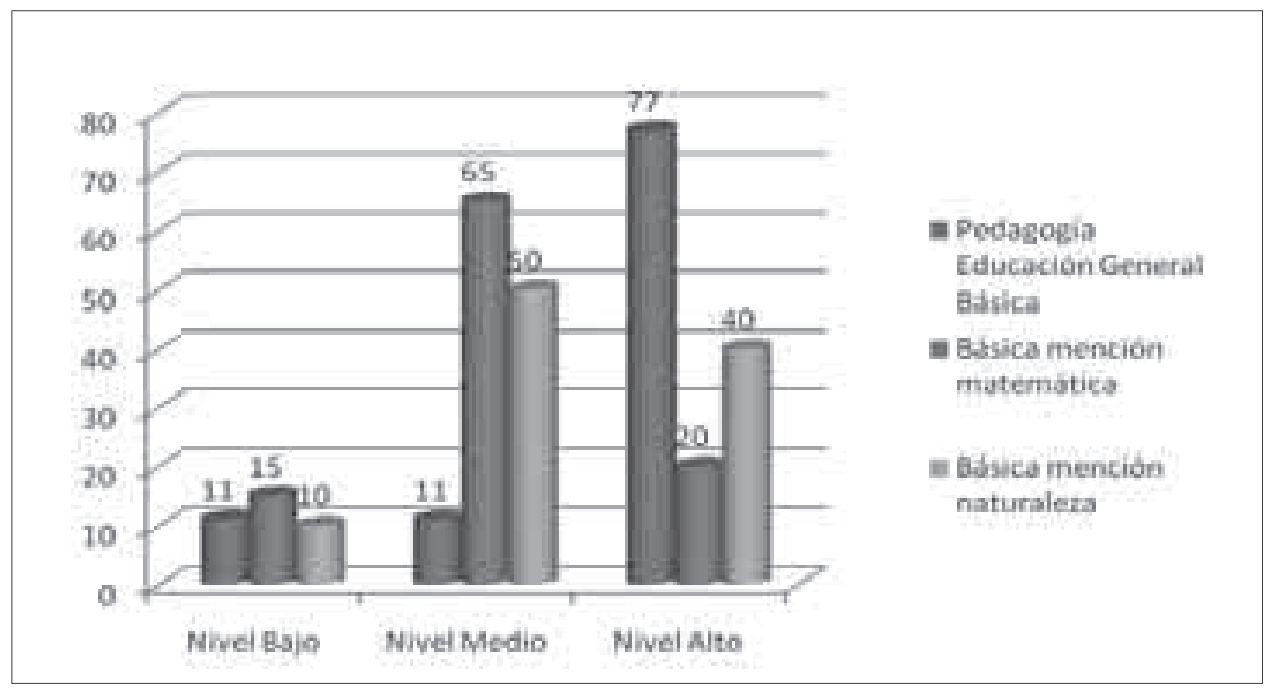

Las reflexiones científicas tecnológicas de los alumnos de pedagogía general básica alcanzan el nivel alto, situándolo en un $77 \%$. Respecto de los alumnos de la Carrera de Básica, con mención en matemática, su reflexión científica tecnológica es un $65 \%$, situándolos en el nivel medio. En la Carrera de Básica mención ciencias de la naturaleza, la reflexión alcanza un $50 \%$, situándolo, en el nivel medio. Al observar los datos de las tres carreras, se puede concluir que los alumnos de la carrera de Educación General Básica alcanzan el nivel más alto de reflexión científica tecnológica.

Gráfico 2. Metodología de proyecto aplicada por los profesores

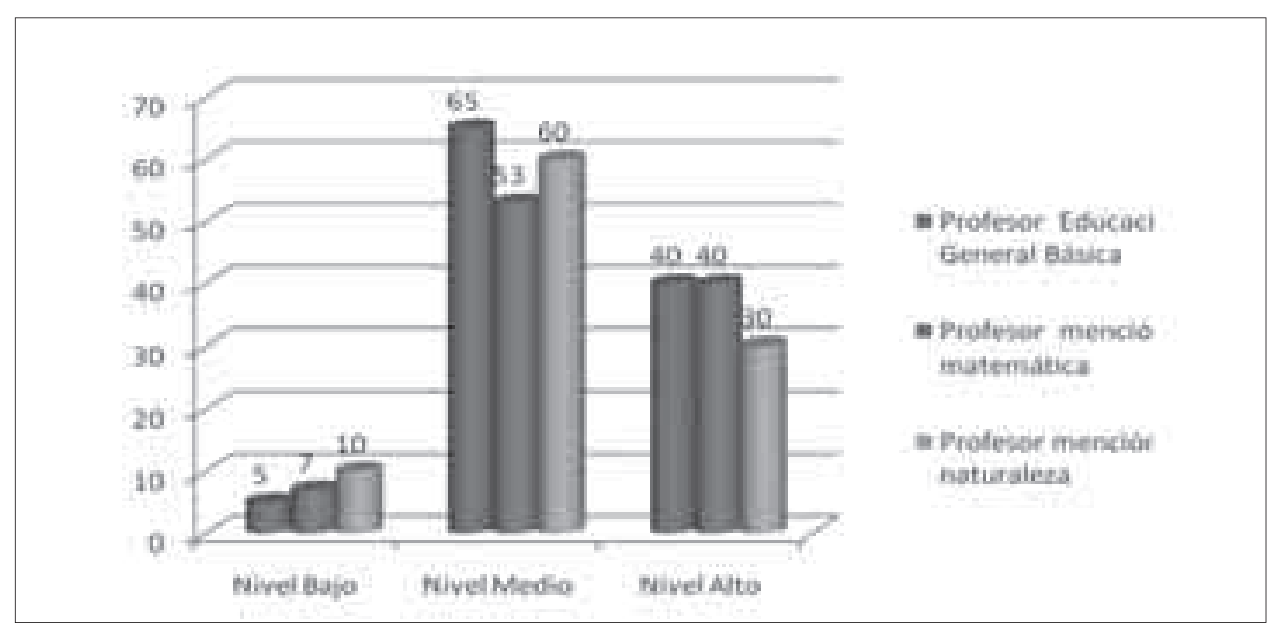


Aplicación de la metodología de proyecto por los docentes en la enseñanza de educación tecnológica, herramienta fundamental para la reflexión científica-tecnológica

La utilización de la metodología de proyecto en las distintas carreras es la siguiente: en Pedagogía General Básica alcanza un $65 \%$ en el nivel medio y un $40 \%$ en el nivel alto. En cuanto a la Carrera de Pedagogía Básica con mención en matemática es un $53 \%$ en el nivel medio, y de un $40 \%$ en el nivel alto. Respecto de la Carrera de Pedagogía Básica con mención en ciencias de la naturaleza, esta alcanza un $60 \%$ en el nivel medio, y un $30 \%$ en el nivel alto. Queda de manifiesto que a mayor utilización de la metodología de proyecto, mayor es el desarrollo de la reflexión científica-tecnológica. La didáctica que implementa el profesor afecta positivamente en la reflexión científica-tecnológica de los alumnos de estas carreras universitarias. Los autores señalados en el marco teórico manifiestan que esta metodología, presenta ese componente importante de reflexión sobre el hacer. Obtener información y analizarla es un proceso fundamental en esta metodología. gráficos.

\section{Discusiones e implicaciones futuras}

A continuación se presentan los principales hallazgos y su discusión derivada de este estudio. La educación tecnológica es una disciplina relativamente nueva y muy transversal, e integradora de conocimientos de distintas asignaturas del aprendizaje, una de las preguntas que se debería hacer es: ¿Es posible implementar políticas educativas para profundizar su desarrollo e implementación a nivel nacional en forma óptima, incluso en los niveles de otras carreras?

La educación tecnológica ha sido asumida en las distintas universidades, muchas veces por profesores que no están capacitados para desarrollar en forma óptima esta asignatura. Es conveniente preguntarse: $i$ Es posible que si se trabaja con profesores formados en la universidad, especialistas en Educación Tecnológica, esto permita mejores aprendizajes científicos-tecnológicos y en consecuencia repercuta positivamente en el SIMCE? ${ }^{5}$ Por otro lado, la formación de conciencia ciudadana en el uso de las energías limpias y profundización en los conceptos de innovación y emprendimiento; trabajo en equipo y solución de problemas, se hace cada vez más urgente y necesario. De esta manera, esta visión se ajusta a los requerimientos de la Educación Tecnológica en Chile y sus metodologías.

Finalmente, ¿Qué tan importante es esta metodología en la forma de abordar la educación tecnológica? En los análisis realizados y en la triangulación con el marco teórico y los autores señalados en la bibliografía, se percibe una fuerte vinculación de esta metodología con los resultados que queremos de nuestros alumnos, en cuanto a

5 Sistema medición de la calidad de la educación en Chile. Este sistema está incorporado en todos los niveles de la enseñanza básica y media del país. Especialmente en las asignaturas del área científica. Aunque en los últimos años se ha estado incorporando mediciones en otras asignaturas del currículo nacional. En la actualidad este tipo de medición se encuentra muy cuestionado. 
la reflexión sobre el hacer científico-tecnológico. Además, el estudio señala que con otras metodologías es menos probable que se pueda lograr resultados esperados como se puede deducir en los resultados de las otras carreras incorporadas en el estudio. La metodología de proyecto es conducente a un "hacer reflexivo, científico- tecnológico".

\section{Conclusiones}

Queda de manifiesto que cuando las prácticas pedagógicas son pertinentes al marco curricular y de acuerdo con el método propio de la asignatura, el desarrollo de la reflexión científica-tecnológica es mayor, como se concluye en la variable Pedagogía General Básica. Esto tiene que ver con la organización de los espacios y de los tiempos en el protagonismo de los alumnos en el desarrollo de la actividad científica-tecnológica. Los alumnos de la carrera de Educación General Básica han tenido Educación Tecnológica prácticamente durante seis semestres de ocho que dura la Carrera.

En los demás resultados de este estudio, el grado de reflexión científica-tecnológica de los alumnos de las demás carreras, como Pedagogía con mención en matemática y mención ciencias, presentan un nivel medio en sus reflexiones, lo cual significa que se requiere acciones permanentes y duraderas respecto de la utilización de la metodología en estudio.

En cuanto a las metodologías utilizadas, se puede concluir que es necesario avanzar a un mayor desarrollo de la metodología en estudio. Ya que solamente su utilización alcanza un nivel medio. Además, se pudo identificar que se utilizan metodologías tradicionales, tales como conducción reiterada y exposición oral del profesor. La Metodología estudiada entrega elementos necesarios para innovar y mejorar la práctica pedagógica. Entre ellas, la reflexión respecto de situaciones problemáticas, el análisis de las mismas situaciones, la búsqueda de información y la constate evaluación de las mejores respuestas al problema presentado.

En muchas de las repuestas de los profesores se pudo detectar que las actividades que se realizan no están diseñadas a partir de la metodología de proyecto. Acciones sueltas y sin sentidos; falta más enseñanza interdisciplinaria y conectada con el mundo real más allá del aula. Las características metodológicas que se utilizan en estas carreras (Básica con mención en matemática y de las ciencias de la naturaleza), presentan tendencias tradicionales-conductistas.

Se hace necesario reorientar las necesidades de un fuerte apoyo para enfrentarse a un mundo cada vez más complejo en la enseñanza y en el aprendizaje de los alumnos. Es inevitable que el profesor de ciencia y tecnología adquiera las competencias metodológicas que le permita guiar y orientar a los estudiantes hacia un aprendizaje significativo, pertinente, duradero, y que le sirva para la vida. 


\section{Referencias}

Branda L.A. (2008). El aprendizaje basado en problemas. El resplandor tan brillante de otros tiempos. En U.F. Araújo y G. Sastre (Eds.). El aprendizaje basado en problemas. Una nueva perspectiva de la enseñanza en la universidad. (pp. 1746). Barcelona: Gedisa.

Bradbury L.U. (2010). Educative Mentoring: Promoting reform-based science teaching through mentoring relationships. Science Education. 94, 1049-1071.

Briones, G. (1994). Instrumentos de evaluación de proyecto: iniciación a la vida y la creatividad, Santiago: Ediciones: CPEIP.

Cajas, F. (2001). Alfabetización científica y tecnológica: la transposición didáctica del conocimiento tecnológico. Enseñanza de las ciencias. 19 (2), 243-254.

Díaz, B. F. (2006). Concepción actual de la estrategia de proyectos y competencias que promueve. En Enseñanza situada. Vínculo entre la escuela y la vida. México: Mc Graw Hill.

Famiglietti, M. (2001). Didáctica y metodología de la educación tecnológica. Buenos Aires: Ediciónes Homo Sapiens.

Ferrer, G. (1983). Le traject de la formation. Les enseignants entre la théorie et la pratique. En González S. M. (1995). Formación docente: perspectivas desde el desarrollo del conocimiento y la socialización profesional. Barcelona: PPU.

Ferrero, M. (2001). El área de tecnología, aportes para su implementación y perspectivas de desarrollo en los centros educativos. Argentina: Editorial Homo Sapiens

Ferreira, C., Gil-Pérez, D. y Vilches, A. (2006). Imagen de la tecnología transmitida por los textos de educación tecnológica. Didáctica de las Ciencias Experimentales y Sociales. 20, 23-46.

Gutiérrez, M. (2002). Tecnología /l educación media. Santiago de Chile: Ediciones. Santillana S.A.

Hernández, S. (1999). Metodología de la investigación, (2. ${ }^{a}$ ed.). México: McGraw Hill.

Jacques, G. (2001). ¿Qué metodología para qué educación tecnológica? Santiago: Fernando Mena Ediciones.

Martin, M. y González, J. C. (2002). Reflexiones sobre la educación tecnológica desde el enfoque CTS. Revista Iberoamericana de Educación. 28, 17-59.

Martín, M. y Osorio, C. (2003). Educar para participar en ciencia y tecnología. Un proyecto para la difusión de la cultura científica. Revista Iberoamericana de Educación 32, 165 210.

Mellado, V. (2001). ¿Por qué a los profesores de ciencias nos cuesta tanto cambiar nuestras concepciones y modelos didácticos? Revista Interuniversitaria de Formación del Profesorado. 40, 17-30. 
Muñoz, A., Díaz, M. (2009). Metodología por proyectos en el área de conocimiento del medio. Revista Docencia e Investigación. 19,101-126

Nikel G. (2014). Proyecto de formación Del profesorado universitario de Ciencias, Matemáticas y Tecnología, en las metodologías de Aprendizaje Baade en Problemas y Proyectos. Enseñanza de las Ciencias. 32(2),113-129.

Liston, D.P., Zeichner, K.M. (1991). Formación del profesorado y condiciones sociales de escolarización. La Coruña: Ediciones Morata, S.L.

López, R. (2001). La evaluación en el área de tecnología en secundaria, Madrid: Editorial Narcea

Oyarzún, N. (2006). Educación tecnológica el buen emprendedor y desarrollo de competencias, Universidad de Los Lagos. Osorno. Editorial Universidad De Los Lagos, Chile.

Pavié, A. (2011). Formación docente: hacia una definición del concepto de competencia profesional docente, REIFOR. Revista Interuniversitaria de Formación del Profesorado.. $14(1), 67-80$

Pérez G. A. (1998). La cultura escolar en la sociedad neoliberal. Madrid: Morata.

Pinto, R. (2001). Mejoramiento de la calidad de los aprendizajes en el marco de la reforma educacional chilena. En Análisis y desafíos de la reforma educacional chilena. Santiago: Ministerio de Educación/FIDE.

Pozuelos, F. (2007). Trabajo por proyectos en el aula: descripción, investigación y experiencias. Sevilla: Cooperación Educativa.

UFAP. Aprendizaje basado en problemas. Disponible en: http://ufap.dgdp.uaa.mx/descargas/ abp_aprendizaje.pdf Consultado: Julio 7 Abril, 2015

Recibido: 2 de abril 2014

Aceptado: 14 de agosto 2014

Como citar:

Oyarzún, N. (2015) Aplicación de la metodología de proyecto por los docentes en la enseñanza de educación tecnológica, herramienta fundamental para la reflexión científica-tecnológica. Praxis Pedagógica, 16, 75-92. 